\title{
Fgf2 is expressed in human and murine embryonic choroid plexus and affects choroid plexus epithelial cell behaviour Sarah Greenwood ${ }^{1,4}$, Adam Swetloff 1,5 , Angela M Wade ${ }^{2}$, Tetsuya Terasaki ${ }^{3}$ and Patrizia Ferretti*1
}

\begin{abstract}
Address: ${ }^{1}$ DevelopmentalBiology Unit UCL Institute of Child Health, 30 Guilford Street, London WC1N 1EH, UK, ${ }^{2}$ Paediatric Epidemiology \& Biostatistics, UCL Institute of Child Health, 30 Guilford Street, London WC1N 1EH, UK, ${ }^{3}$ Membrane Transport and Drug Targeting Laboratory, Graduate School of Pharmaceutical Sciences, Tohoku University, Aoba, Aramaki, Aoba-ku, Sendai 980-8578, Japan, ${ }^{4}$ Department of Applied Biological Sciences, School of Community and Health Sciences, City University, 20 Bartholomew Close, London EC1A 7QN, UK and ${ }^{5}$ Department of Genetic Medicine and Development, University of Geneva, 1 Rue Michel-Servet 1211 Geneva-4, Switzerland

Email: Sarah Greenwood - s.greenwood@city.ac.uk; Adam Swetloff - Adam.Swetloff@medecine.unige.ch; Angela M Wade - a.wade@ich.ucl.ac.uk; Tetsuya Terasaki - terasaki@mail.pharm.tohoku.ac.jp; Patrizia Ferretti* - ferretti@ich.ucl.ac.uk

* Corresponding author
\end{abstract}

Published: 29 December 2008

Cerebrospinal Fluid Research 2008, 5:20 doi:10.1186/1743-8454-5-20
Received: 23 September 2008

Accepted: 29 December 2008

This article is available from: http://www.cerebrospinalfluidresearch.com/content/5/I/20

(c) 2008 Greenwood et al; licensee BioMed Central Ltd.

This is an Open Access article distributed under the terms of the Creative Commons Attribution License (http://creativecommons.org/licenses/by/2.0), which permits unrestricted use, distribution, and reproduction in any medium, provided the original work is properly cited.

\begin{abstract}
Background: Although fibroblast growth factor (Fgf) signalling plays crucial roles in several developing and mature tissues, little information is currently available on expression of Fgf2 during early choroid plexus development and whether Fg2 directly affects the behaviour of the choroid plexus epithelium $(\mathrm{CPe})$. The purpose of this study was to investigate expression of Fgf2 in rodent and human developing $\mathrm{CPe}$ and possible function of $\mathrm{Fg} 2$, using in vitro models. The application of $\mathrm{Fg} 22$ to brain in vivo can affect the whole tissue, making it difficult to assess specific responses of the $\mathrm{CPe}$.

Methods: Expression of Fg2 was studied by immunohistochemistry in rodent and human embryonic choroid plexus. Effects of Fgf2 on growth, secretion, aggregation and gene expression was investigated using rodent $\mathrm{CPe}$ vesicles, a three-dimensional polarized culture model that closely mimics $\mathrm{CPe}$ properties in vivo, and rodent $\mathrm{CPe}$ monolayer cultures.
\end{abstract}

Results: Fgf2 was present early in development of the choroid plexus both in mouse and human, suggesting the importance of this ligand in Fof signalling in the developing choroid plexus. Parallel analysis of Fgf2 expression and cell proliferation during CP development suggests that Fgf2 is not involved in $\mathrm{CPe}$ proliferation in vivo. Consistent with this observation is the failure of Fgf2 to increase proliferation in the tri-dimensional vesicle culture model. The CPe however, can respond to Fgf2 treatment, as the diameter of $\mathrm{CPe}$ vesicles is significantly increased by treatment with this growth factor. We show that this is due to an increase in cell aggregation during vesicle formation rather than increased secretion into the vesicle lumen. Finally, Fgf2 regulates expression of the CPe-associated transcription factors, Foxjl and E2f5, whereas transthyretin, a marker of secretory activity, is not affected by Fof2 treatment.

Conclusion: Fgf2 expression early in the development of both human and rodent choroid plexus, and its ability to modulate behaviour and gene expression in $\mathrm{CPe}$, supports the view that Fgf signalling plays a role in the maintenance of integrity and function of this specialized epithelium, and that this role is conserved between rodents and humans. 


\section{Background}

The choroid plexus epithelium (CPe) is a specialized neuroepithelium that is involved in secretion of cerebrospinal fluid (CSF) into the cerebral ventricles and in maintaining the homeostasis of the brain during development and throughout life [1-3]. Most CSF is secreted by the CPe and is re-absorbed mainly at the site of the arachnoid villi. Knockout of genes expressed in the CPe, such as E2f5, a member of a family (E2f1-E2f6) of transcriptional regulators, FoxJ1, a member of the forkhead-box (Fox)/winged helix gene family, and $p 73$, have been associated with hydrocephalus [4-6]. The mechanisms leading to the apparently non-obstructive hydrocephalus in these mutants appear to differ and have yet to be fully elucidated. These genes are expressed early during CPe development in rodent and humans [6-11] and Foxj1 has been reported to be down-regulated by Fgf2 (fibroblast growth factor 2) in neural cells [12].

Some biogenic amines, neuropeptides and hormones also have the ability to modulate the function of the CPe, including its secretory activity [13-17], but the effect of growth factors on its cellular function has not been well studied, especially in the embryonic CPe. Fgf2 has been implicated in the regulation of cell survival and apoptosis, adhesion, motility, and differentiation [18], and in the brain, among other effects, in the control of neural stem cell proliferation both during development and in the adult following intraventricular administration. It has also been suggested that Fgf treatment can stimulate neurogenesis and aid repair following brain injury [19-21]. Injection of Fgf2 into the ventricles of the brain, however, has been reported to induce hydrocephalus [22-24]. This could be a hindrance in the development of Fgf-based treatments [25], as the mechanisms underlying the effect of Fgf2 on CSF accumulation remain unclear. Given that Fgf can affect several cell types in the brain, induction of hydrocephalus following Fgf administration might not be due to a direct effect of Fgf on the CPe. On the other hand, Fgf2 might directly affect CSF secretion or reabsorption [23] or even CSF circulation. For example, defects in ciliogenesis, as reported in the FoxJ1 knock-out mouse, can affect CSF dynamics in the ventricles and result in hydrocephalus [5].

As members of the fibroblast growth factor receptor family (FgfR 1-4) are expressed in the CPe and are differentially regulated during development [26], it is likely that Fgf signalling can directly affect at least some aspects of CPe cell behaviour. While in vitro models cannot reproduce the complexity of the responses leading to the hydrocephalic phenotype in vivo, they can allow the direct effects of Fgf on CPe cells to be tested.
At least one FgfR ligand, Fgf2, is known to be expressed in the adult and foetal CPe [27-29], but its expression during early development has not been investigated either in rodents or humans. We have therefore assessed Fgf2 pattern of expression during mouse and human CP development in relation to proliferation. Having found that Fgf2 protein is expressed both in human and mouse embryonic choroid plexus, we have used embryonic CP cells cultured in Matrigel to investigate putative roles of Fgf2 on $\mathrm{CPe}$ behaviour. In this tri-dimensional matrix, embryonic CP cells form fluid-filled spherical structures called vesicles, and we have recently shown that their growth depends on secretion within the lumen [30,31]. The CPe phenotype and the behaviour of the cells in this culture system appear to be very similar to that in vivo, representing a valuable model to assess the effect of growth factors on cell-cell adhesion and cell migration as well as fluid secretion and solute transport [30]. This is further supported by this study, as we show that Fgf2 does not affect $\mathrm{CPe}$ proliferation in vitro in a tri-dimensional environment, consistent with the significant Fgf2 expression and limited proliferation observed in the choroid plexus in vivo. We also show that whereas Fgf2 treatment in vitro affects CPe vesicle formation in a dose-dependent fashion, it does not alter its secretory activity. Fgf2, however, can regulate expression of the $\mathrm{CPe}$-associated transcription factors E2f5 and FoxJ1. Altogether this study suggests that Fgf signalling plays a role in CPe development and possibly in its integrity and homeostasis.

\section{Methods}

\section{Tissue collection and reagents}

Choroid plexus for cultures, and brains to be fixed in $4 \%$ paraformaldehyde and paraffin embedded for immunohistochemistry were dissected from out-bred CD-1 albino mice supplied by Charles River Mouse Farms (Kent, UK) and housed under Home Office regulations. The morning of detection of a vaginal plug was designated as embryonic day 0.5 (E0.5). Mice were killed by cervical dislocation according to Home Office regulations at the times indicated in the results section and embryos removed for immunohistochemical analysis or CPe culture preparation as described below. Paraffin sections from human embryos at 8 weeks of gestation obtained under ethical approval were provided by the Wellcome/MRC-funded Human Developmental Biology Resource. Unless otherwise specified, reagents used were from Sigma-Aldrich, UK.

\section{Choroid plexus cell cultures}

The fourth ventricle CP from E12.5 mice was dissected and CP cells isolated and cultured as previously described [30,31]. Cells were grown in HEPES-buffered Dulbecco's modified essential medium (H-DMEM, Gibco-BRL, Paisley, UK) supplemented with $10 \%$ foetal calf serum (FCS) 
and penicillin-streptomycin (Gibco-BRL) either as monolayers on laminin (Sigma, UK) or seeded in Matrigel (Becton Dickenson, distributed by Stratech Scientific Ltd, Dudley, UK) to allow vesicle formation. In vesicle culture experiments $1-2 \times 10^{5} \mathrm{CPe}$ cells were plated on to Matrigel in cloning cylinders of $8 \mathrm{~mm}$ diameter and fed every 2-3 days with the culture medium described above.

The TRCSFB- 2 cell line was established from CPe cells isolated from a transgenic rat harboring the temperature-sensitive simian virus 40 (ts SV 40) large T-antigen gene [32]. Cells were grown in DMEM (Gibco-BRL) supplemented with $10 \%$ FCS and penicillin-streptomycin on collagen I (Vitrogen, Cohesion Technologies, Inc. CA, USA) at $33^{\circ} \mathrm{C}$ [32]. Before carrying out RNA expression studies, TRCSFB2 cells were cultured for $24 \mathrm{~h}$ at $37^{\circ} \mathrm{C}$.

\section{Cell analysis and treatments}

CPe behaviour was monitored by regular observation under an inverted microscope (IM45 Zeiss) and by timelapse photography for up to 4 consecutive days using Openlab software (Improvision, UK). For Fgf2 treatment, Fgf2 (R\&D Systems, Oxford, UK) at the desired concentration was added to the culture medium either at the time of plating CPe cells or later as specified in the Results. To study the effect of Fgf2 on vesicle recovery after inhibition of secretion and collapse, cultures were treated for $48 \mathrm{~h}$ either with a combination of $100 \mu \mathrm{M}$ acetazolamide and $5 \mathrm{mM}$ ouabain or with the vehicle alone (DMSO controls).

\section{Proliferation analysis and immunohistochemistry}

Fgf2 was detected by an anti-Fgf2 mouse monoclonal antibody (Transduction Laboratories, Lexington, UK, 1:100 dilution). Cell proliferation was detected by: antibromo-deoxyuridine (BrdU) rat monoclonal antibody to detect cells in $S$ phase that had incorporated BrdU (Oxford Biotechnology, Oxford, UK, 1:100); anti-phosphorylated-histone 3 (p-H3) rabbit antibody to detect cells in $M$ phase (Upstate Biotechnology, Buckingham, UK; 1:100 or 1:500 dilution, see below); a mouse monoclonal antibody to PCNA, an auxiliary factor for DNA polymerase delta, to detect cells in G1-S phase (clone PC10, Santa Cruz Biotechnology, Inc., CA, USA, 1:100). The secondary antibodies used, fluorescein-conjugated goat anti-rabbit (1:50 dilution), and peroxidase-conjugated goat anti-rabbit, goat anti-mouse and goat anti-rat immunoglobulins (1:100 dilution), were all from DAKO (Bucks, UK). Hoechst dye H33258 (1:500 dilution of a $1.2 \mathrm{mg} / \mathrm{ml}$ stock) was used to counter-stain nuclei.

Mitotic cells in CPe vesicles and aggregates were detected using the anti-p-H3 antibody. Cells grown in Matrigel inside cloning cylinders placed onto glass coverslips were fixed in $4 \%$ paraformaldehyde (PFA) for $10 \mathrm{~min}$. Non- specific binding sites were blocked with $1 \%$ goat serum in PBS for $40 \mathrm{~min}$. CPe vesicles/aggregates were incubated overnight at $4{ }^{\circ} \mathrm{C}$ with the anti-p-H3 antibody, thoroughly washed and incubated with fluorescein-conjugated goat anti-rabbit for $30 \mathrm{~min}$. Nuclei were counterstained for 15 min with Hoechst dye.

The anti-PCNA monoclonal antibody was used to stain primary CPe cells fixed for $10 \mathrm{~min}$ in 4\% PFA. Bound antibody was detected using a fluorescein-conjugated goat anti-mouse antibody and nuclei counterstained with Hoechst dye. Paraffin sections were stained either with the anti-Fgf2 mouse monoclonal antibody or with the anti-pH3 rabbit antibody (1:500 dilution) essentially as previously described $[7,26]$.

Tissues and cells were viewed under a Zeiss Axioplan microscope and digitally scanned using a Hamamatsu digital camera (C4742-95, Hamamatsu Photonics KK, Japan) into Openlab software (Improvision Ltd, Coventry, UK).

\section{RT-PCR}

TRCSFB- 2 cells were trypsinised and the pellet stored in 1 $\mathrm{ml}$ TRI-Reagent at $-20^{\circ} \mathrm{C}$ until RNA extraction following the manufacturer's protocol. cDNA was synthesised from $1 \mu \mathrm{g}$ of RNA using M-MLV reverse transcriptase and random hexamers according to the manufacturer's instructions (Promega, Southampton, UK). The PCR step was performed using Taq polymerase at an annealing temperature of $57^{\circ} \mathrm{C}$ with specific primer pairs for each gene, which were designed to span intron-exon boundaries to exclude genomic DNA contaminations. The linearity range of amplification for each set of primers was evaluated and the number of cycles selected accordingly. The primers (Genosys, Cambridge, UK) used and number of cycles and product size (in brackets) are: Gapdh $(20,491$ bp) 5' TTCCAGTATGACTCCACTCACG 3', 5' GGATGCAGGGATGATGTTCT 3'; E2f5 (25, 227 bp) 5' TGTGGCTACAGCAAAGCATC 3', 5' GGCCCTGAGTGACTCTTCAG 3'; FoxJ1 (25, 205 bp) 5' TACTGCTGACCCAGGAGGAG 3', 5' GGTAGCAGGGCAGTTGATGT 3', TTR (30, 505 bp) 5' CAGATCCACAAGCTCCTGAC 3', 5' CTGCTTTGGCAAGATCCTGC 3'; p73 (30, 253 bp) 5' AGAGTGTGGTTGTGCCGTATG 3', 5' TCCCGGTAATGGTCTTCATC 3'. PCR products were visualized on a $1.5 \%$ agarose gel containing ethidium bromide under ultraviolet light and imaged by using a Gel imaging system and Alphaease 3.3 software (GRI Ltd, Braintree, UK).

\section{Measurements and statistical analysis}

The diameter of each vesicle was measured using an eyepiece graticule at $\times 20$ magnification. Measurements were taken to within the nearest quarter graduation on the eyepiece. Each vesicle could be identified easily by recording 
the co-ordinates on a finely graduated $\mathrm{x}-\mathrm{y}$ stage and was measured at 2-day intervals at approximately the same time of day on days $4,6,8$, and 10 . The diameters were recorded 'blind' to treatment group.

The number and size of aggregates and vesicles was measured using the Openlab 3.1.5 software. The size of aggregate and vesicles was estimated by measuring the surface area in $\mu \mathrm{m}^{2}$ of their focal equatorial plane. The measurements were automatically recorded by the Openlab 3.1.5 programme, stored in Microsoft Excel and later transferred to SPSS Statistics 11.5 (SPSS Inc.) for statistical analysis. Aggregates and vesicles were measured in the same fields at different days. Four fields of view were taken on each measurement day. Cell motility was assessed following time-lapse photography by measuring the distance travelled by a cell using the Openlab 3.1.5 software.

Data are expressed as means \pm standard error of the mean (mean \pm SEM). Parametric and non-parametric tests as appropriate were used to compare treatment groups at different time points. Regression analysis was used to quantify the effects of treatments over time when different vesicles were assessed on each day. Multilevel modelling was used to model the serial changes in individual vesicles over time [33]. Results are presented with $95 \%$ confidence intervals.

\section{Results \\ Expression of Fgf2 and the mitotic marker, $\mathrm{p}-\mathrm{H} 3$ in developing $\mathrm{CPe}$}

Previously, expression of Fgf2 in the CPe has not been studied at early developmental stages, nor across species. Therefore, this study investigated whether Fgf2 is present in the early embryonic mouse and human CP by immunohistochemistry. In the E12.5 mouse IVth ventricle $\mathrm{CP}$, Fgf2 protein was strongly expressed in the epithelium and more weakly in the mesenchyme (Fig. 1A-B), and its expression was maintained at E16.5 (not shown). High levels of Fgf2 protein were also detected in both IVth and lateral ventricle $\mathrm{CPe}$ of human embryos at 8 weeks of gestation (Fig. 1C-E). To establish whether Fgf2 is expressed in dividing CP cells, we analysed E12.5 mouse IV and lateral ventricle CPe using the mitotic marker, p-H3 (phosphorylated histone 3 ). Very rarely $\mathrm{p}-\mathrm{H} 3$ staining was observed in IVth and lateral ventricle CPe, whereas cells of the ventricular zone were frequently labelled (Fig. 1F-G). Some p-H3 positive cells were observed in the CP stalk of the IV ventricle (Fig. 1G). The noticeable difference in the expression patterns of Fgf2 and p-H3 suggests that the main role of Fgf2 during CPe development is not the control of CP cell proliferation.

\section{Effect of FGF2 on CPe proliferation and vesicle diameter} As the CPe expresses Fgf2 and FgfRs, and at least FgfR2 is detected in CPe vesicles ([26] and unpublished observation), we further investigated the effect of Fgf2 on cell proliferation in primary CPe cells grown as monolayers and in $\mathrm{CPe}$ vesicles. We first studied the effect of different doses of Fgf2 on the proliferation of TRCSFB-2 cells (data not shown). Fgf2 significantly increased anti BrdU incorporation at 1 and $10 \mathrm{ng} / \mathrm{ml}$ compared to controls $(p<$ 0.001 ). However, with increasing dose of Fgf2 the stimulation of cell proliferation declined and at the highest Fgf2 concentration tested $(100 \mathrm{ng} / \mathrm{ml})$ no difference in TRCSFB-2 proliferation was observed compared to untreated controls. Similarly, $10 \mathrm{ng} / \mathrm{ml}$ Fgf2 significantly increased cell proliferation in primary embryonic CPe cells grown as monolayers ( $p<0.0001)$ (data not shown). Unlike in CPe monolayers, very few cells in control vesicles were mitotic (Fig. 2A-B), and the effect of $10 \mathrm{ng} / \mathrm{ml}$ Fgf2 on p-H3 expression was not significant either in 1 day cultures (not shown) or in 4 day vesicle cultures (Fig. 2C). Analysis of time lapse photography over 2 to 4 days, either at the time of plating or once vesicles had formed, did not provide any evidence of significant cell proliferation in Matrigel either in the presence or absence of Fgf2. Nonetheless, larger vesicles were observed in Fgf2-treated cultures than in controls (Fig. 3A). To further investigate Fgf2 effects on vesicle size, CPe cells plated in Matrigel were treated with $0.75,7.5 \mathrm{or} 75 \mathrm{ng} / \mathrm{ml} \mathrm{Fgf2} \mathrm{from} \mathrm{the} \mathrm{time}$ of plating, and their diameter monitored over 8 days (Fig. $3 \mathrm{~A}-\mathrm{B}$ ). By day 4 all Fgf2-treated vesicles were significantly larger than controls (Student's t-test $p<0.001$ for every dose), and this effect was already significant at $0.75 \mathrm{ng} /$ $\mathrm{ml}$, the lowest concentration used (Fig. 3B).

Altogether these results demonstrate that while Fgf2 increases vesicle diameter, this is not due to increased proliferative activity of CPe cells. They also show that the behaviour of CPe cells in response to Fgf2 depends on the culture environment, given the difference in proliferative effect in monolayer and 3-dimensional (3D) cultures.

\section{Effect of Fgf2 on CPe vesicle secretion}

Analysis of vesicle growth rate showed that the average rate of increase in diameter assessed between 4 and 10 days in culture was the same for control and Fgf2-treated CPe (Fig. 3B). This suggests that Fgf2 does not increase fluid secretion into the lumen, as it has been shown that vesicle growth rate is mainly due to secretion [30]. In addition, when Fgf2 was added to the cultures at 6 days (Fig. 3C), no significant difference in cell diameter between treated and untreated vesicles was observed at 10 days in culture.

To further confirm that Fgf2 does not principally affect fluid secretion, we studied its effects on vesicle recovery 


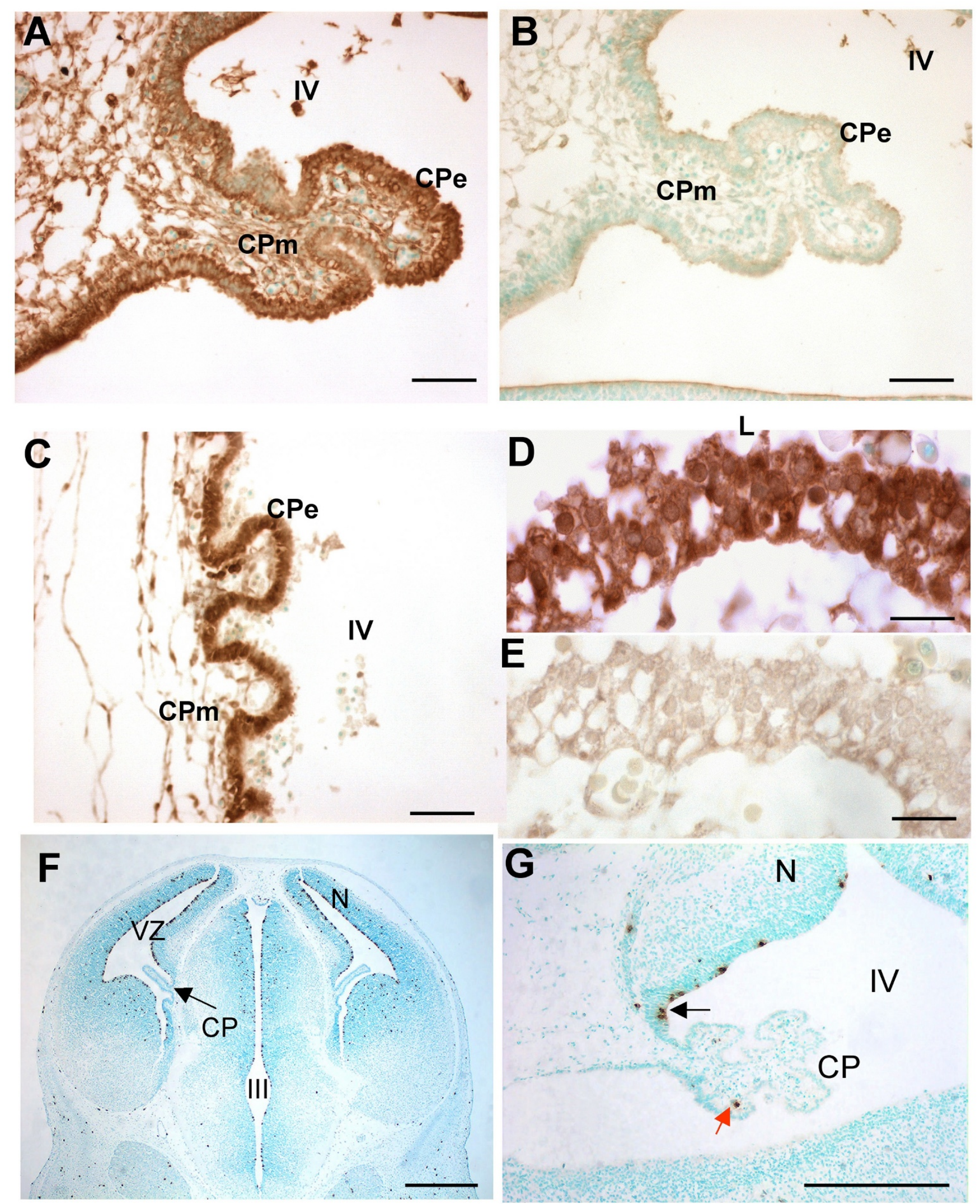

Figure I (see legend on next page) 
Figure I (see previous page)

Expression of Fgf2 and p-H3 in the mouse and human CPe. (A) IV ventricle (IV) choroid plexus epithelium (CPe) in a sagittal section of EI 2.5 mouse embryo brain; positive cytoplasmic staining for Fgf2 protein is observed in the CPe and less strongly in the CP mesenchyme (CPm). (B) Negative control for (A). (C) Human IV ventricle CPe at 8 weeks of gestation: strong Fgf2 labelling is present in the CPe. (D) Human lateral ventricle CPe at 8 weeks of gestation; Fgf2 staining is mainly cytoplasmic. (E) Negative control for (D). (F) P-H3 staining of coronal section of EI 2.5 mouse embryo whole brain, including the choroid plexuses of the lateral ventricles. Neuroepithelial cells of the ventricular zone are frequently labelled with $\mathrm{P}-\mathrm{H} 3$ antibody whereas $\mathrm{P}-\mathrm{H} 3$ labelling in the CPs is rare. $(\mathrm{G})$ Black arrow indicates $\mathrm{P}-\mathrm{H} 3$ labelled cells in the IV ventricle CP stalk; red arrow indicates a P-H3 labelled cell in the CPm. Scale bars are $50 \mu \mathrm{m}$ in A-B; I5 $\mu \mathrm{m}$ in C-D; $500 \mu \mathrm{m}$ in F and $200 \mu \mathrm{m}$ in G. CP = choroid plexus, $\mathrm{CPe}=$ choroid plexus epithelium, $\mathrm{CPm}=$ choroid plexus mesenchyme, $\mathrm{L}=$ lateral ventricle, $\mathrm{N}=$ neuroepithelium, VZ = ventricular zone, III = third ventricle, IV = fourth ventricle.

after inducing them to collapse with secretion inhibitors. Vesicles were cultured for eight days, either with or without $10 \mathrm{ng} / \mathrm{ml}$ Fgf2, and then treated for two days with 5 $\mathrm{mM}$ ouabain and $100 \mu \mathrm{M}$ acetazolamide. The effect of Fgf2 on the recovery in size of collapsed vesicles was then assessed by measuring their area daily over eight days (Fig 4 ). Since the original vesicles were of variable size, the recovery of each vesicle was expressed as percentage of its area before addition of ouabain and acetazolamide (\% recovery). The recovery rates of the vesicles were compared between the two groups using a multilevel model (which accounted for the within vesicle correlation of repeat measurements). The average percentage increase in control vesicle size at each day was 1.19 times higher (95\% confidence interval: $1.17,1.20)$ than the previous day ( $\mathrm{p}<0.05)$. Fgf2-treated vesicles recovered slightly less rapidly than non-treated vesicles, but the difference between control and Fgf2 treatment groups was not significant. These results suggest that fluid secretion rate is not altered by Fgf 2 treatment, and that the observed increase in vesicle size must be induced through different mechanisms at early stages of vesicle formation.

\section{Effect of Fgf2 on CPe vesicle formation}

Having established that Fgf2 treatment does not affect vesicle size by increasing proliferation or secretion and that its major effect on vesicle size occurs prior to day 4, we investigated the effects of Fgf2 on CPe behaviour at early stages of vesicle formation (Fig 5). To this purpose we treated CPe with $10 \mathrm{ng} / \mathrm{ml} \mathrm{Fgf2,} \mathrm{starting} \mathrm{the} \mathrm{treatment} \mathrm{one}$ day after plating on to Matrigel. This allowed us to check that the cultures were at a similar density before addition of the growth factor, and to control for possible variability between experiments carried out from different preparations. The aggregates in Fgf2-treated cultures appeared already to be larger than controls after one day of treatment ( 2 days in culture), fusing with each other much more frequently (Fig 5A-B). After three to four days the aggregates became hollow and formed vesicles that were larger than those in control cultures. As shown by time-
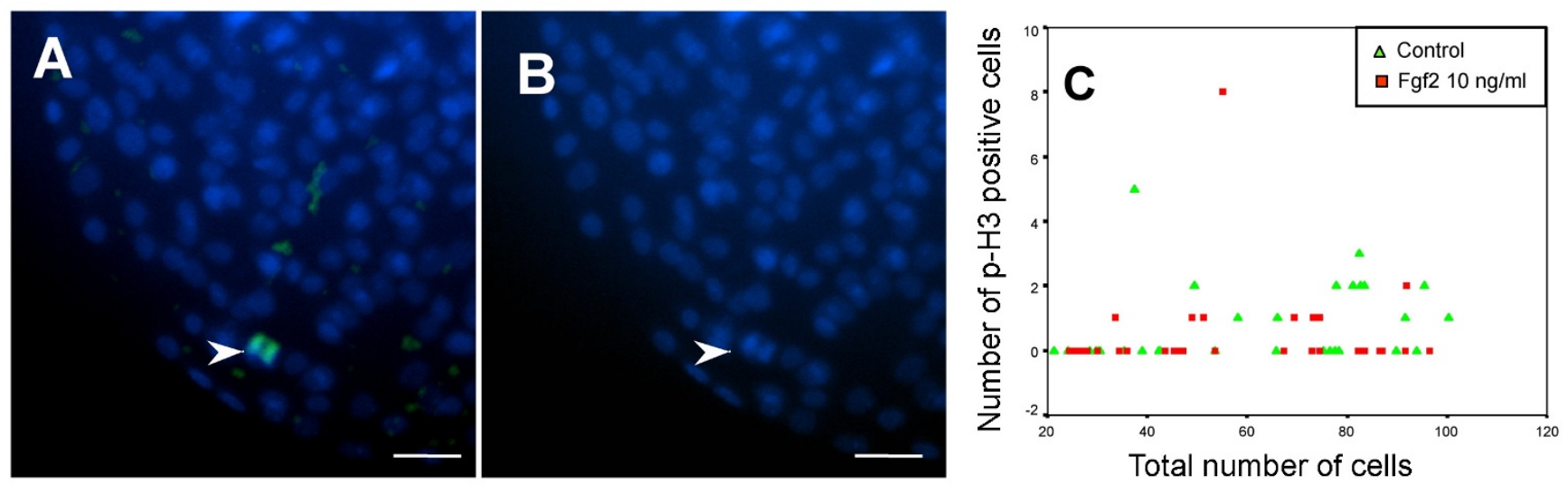

Figure 2

Effect of Fgf2 on cell proliferation in CPe vesicles. (A) Day 4 vesicles were stained for $\mathrm{p}-\mathrm{H} 3$ and nuclei counterstained with Hoechst dye; the arrowhead points to a $\mathrm{p}-\mathrm{H} 3$ stained cell. (B) Same field of view with Hoechst stain only. Scale bars: 25 $\mu \mathrm{m}$. (C) Plot of the number of $\mathrm{p}-\mathrm{H} 3$ labelled cells in day 4 vesicles either untreated or treated with $10 \mathrm{ng} / \mathrm{ml}$ Fgf2 against vesicle total cell number. Data are means \pm SEM. 
A

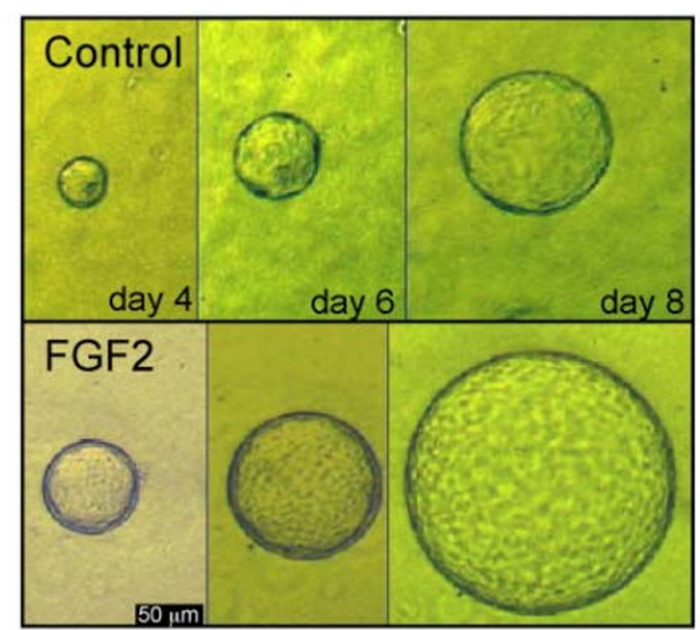

C

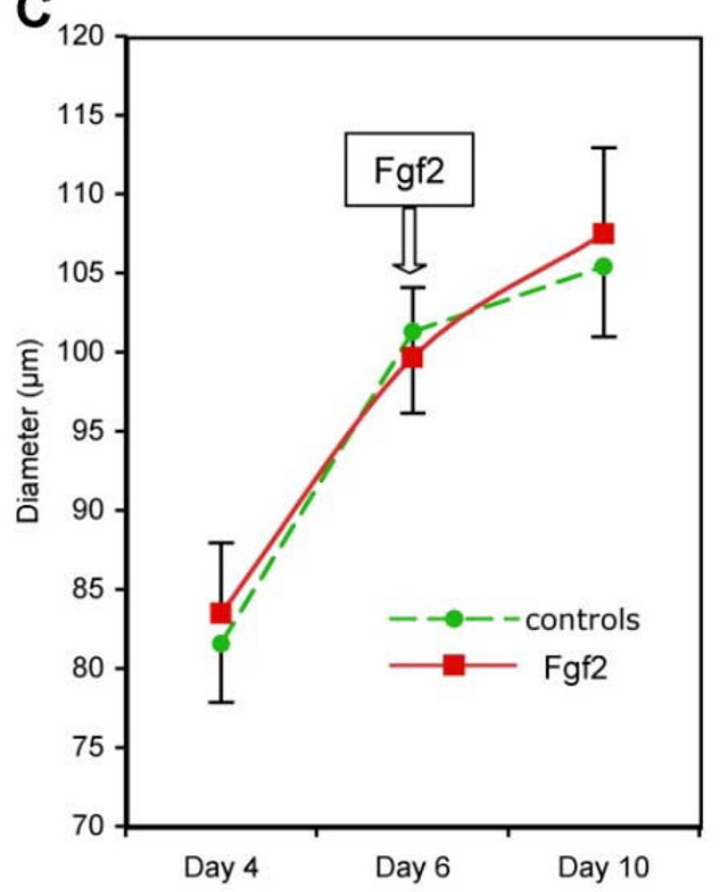

B

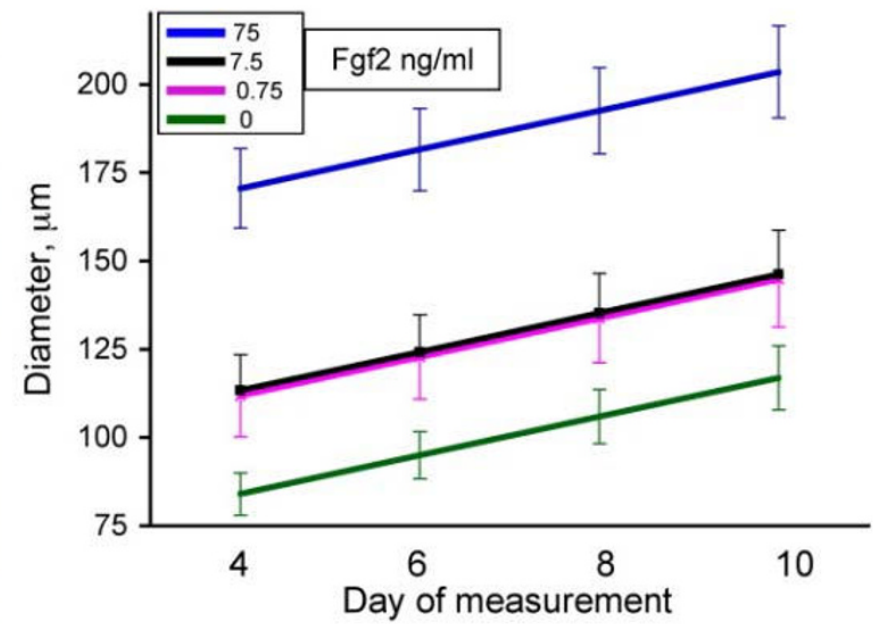

\section{Figure 3}

Effect of Fgf2 on CPe vesicle size and growth. Choroid plexus epithelial cells were cultured in Matrigel either in the presence or absence of Fgf2. (A) Micrographs of two vesicles, one control (upper panel) and one Fgf2-treated (7.5 ng/ml) taken at different times in culture (day 4, day 6 and day 8 ). (B) Graph showing the mean diameters of untreated vesicles (controls, $n=$ $232)$ and vesicles treated with $0.75(n=63), 7.5(n=96)$ or $75(n=54) n g / m l$ Fgf2 measured on days 4, 6, 8 and I0. At all Fgf2 concentrations the vesicle diameter is significantly higher than in controls $(p<0.00 \mathrm{I})$. (C) Graph showing the average vesicle diameters of control vesicles $(n=96)$ and vesicles treated with $10 \mathrm{ng} / \mathrm{ml} \mathrm{Fgf2} \mathrm{from} \mathrm{day} 6$ in culture $(n=110)$. The results were not significant. Data are means \pm SEM. 


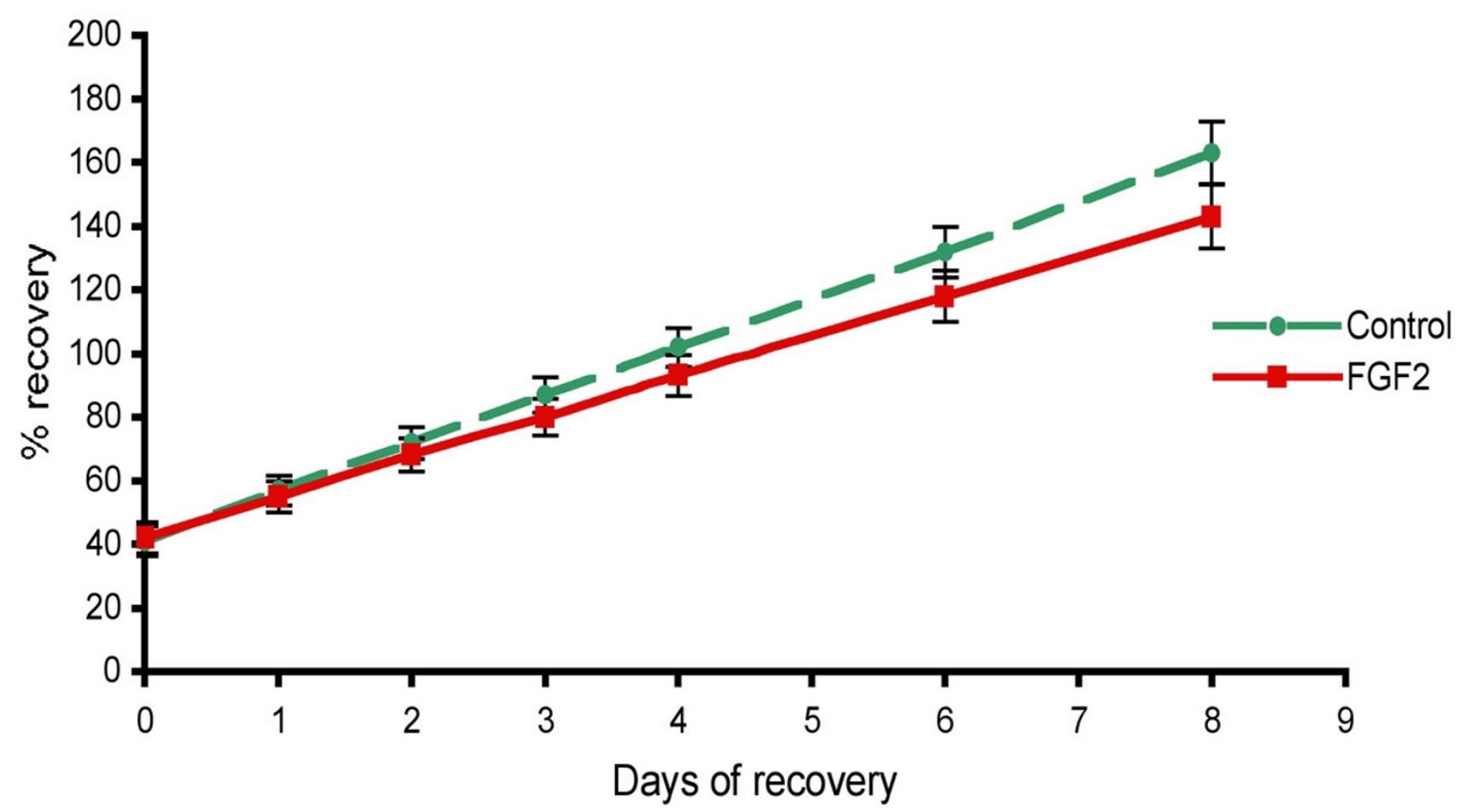

\section{Figure 4}

Effect of Fgf2 on recovery of collapsed vesicles. Graph showing the recovery of collapsed CPe vesicles in response to I0 $\mathrm{ng} / \mathrm{ml}$ Fgf2 expressed as percentage of their size prior to collapse treatment with acetazolamide and ouabain $(\mathrm{n}=\mathrm{I} \mathrm{I} 0$ per group). The extent of recovery between control and Fgf2-treated vesicles was not significantly different. Data are means \pm SEM.

lapse photography, both treated and untreated vesicles were highly pulsatile (Additional files 1 and 2).

In order to quantify the effect of $10 \mathrm{ng} / \mathrm{ml}$ Fgf2, we measured the mid-plane area of CPe aggregates and vesicles in four separate cultures (Fig 5C). Regression analysis revealed that for every day of growth, Fgf2-treated aggregates and vesicles increased in size by an additional $492.03 \mu \mathrm{m}^{2}$ (SEM: 35.72) per day compared to control cultures, and that this effect was highly significant $(\mathrm{p}<$ $0.01)$. We quantified the number of aggregates forming in control and treated cultures, and found that their number was significantly lower in Fgf2-treated cultures than in controls both at one day (two days after plating) and 3 days (4 days after plating) treatment (Fig 5D), as expected given the greater number of cells recruited into each vesicle.

No obvious change in cell motility was observed when cells were treated with $10 \mathrm{ng} / \mathrm{ml}$ Fgf2 at the time of plating. Cell migration was further assessed by measuring the average speed of individual cells in control and $100 \mathrm{ng} / \mathrm{ml}$ Fgf2-treated cultures, to assess whether a change in motility might be more obvious at this higher dose that induces formation of larger vesicles. The speed of cell migration was computed by taking the total distance a cell migrated before incorporation into an aggregate over the amount of time taken to do so. In control cells the average speed was $20.29 \pm 4.84 \mu \mathrm{m} / \mathrm{h}$, and in Fgf2-treated cells the average speed was $20.48 \pm 1.45 \mu \mathrm{m} / \mathrm{h}$. The difference in cell motility was not significant. This suggests that changes in cellcell adhesion, rather than cell motility, are likely to be responsible for the formation of bigger aggregates and consequently larger vesicles in Fgf2-treated cultures.

\section{Fgf2 regulation of genes associated with CPe function}

In order to gain further insight into the possible function of Fgf2 we investigated its effects on the expression of E2f5, FoxJ1, p73 and transthyretin (TTR), a protein synthesized and secreted by the CPe. As the TRCSFB-2 cells appeared to behave in a similar fashion to primary mouse $\mathrm{CPe}$ monolayers when treated with Fgf2 and a large number of these cells can be easily grown, the effect of different concentrations of Fgf2 ( $1 \mathrm{ng} / \mathrm{ml}$, not shown, $10 \mathrm{ng} /$ $\mathrm{ml}$ and $100 \mathrm{ng} / \mathrm{ml}$ ) on gene expression was assessed using semi-quantitative RT-PCR in TRCSFB-2 cells (Fig 6). A consistent reduction ( $\mathrm{p}<0.05)$ in E2f5 and FoxJ1 transcripts was induced by Fgf2 at the highest concentration 

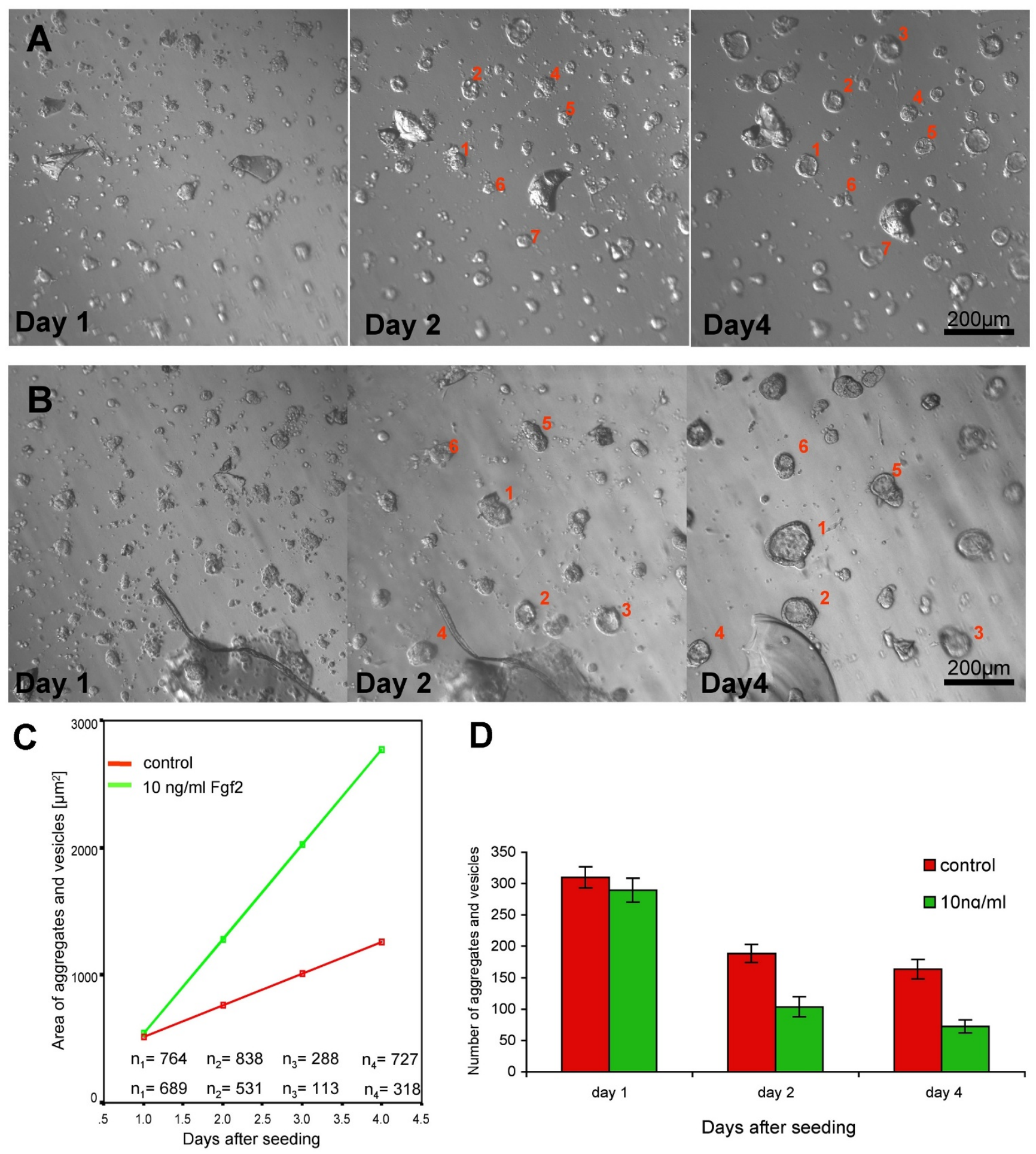

D

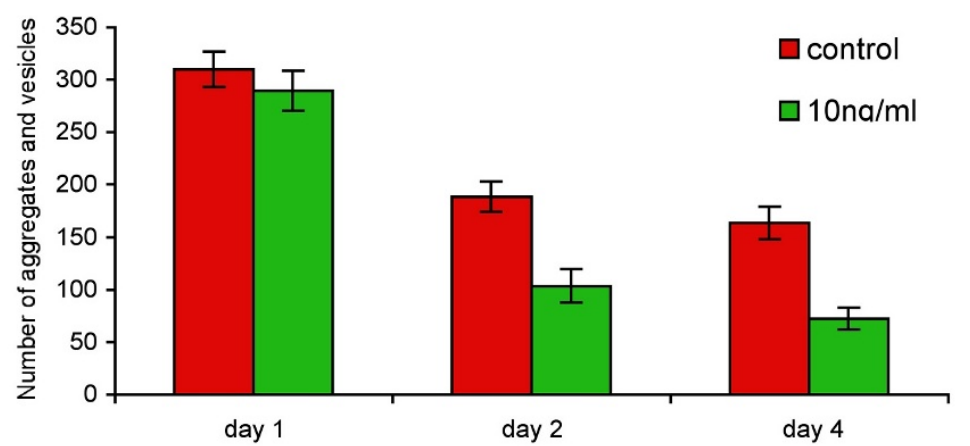

Days after seeding

Figure 5 (see legend on next page) 
Figure 5 (see previous page)

Fgf2 effect on early CPe vesicle formation. (A-B) Early formation of vesicles in the presence of $0 \mathrm{ng} / \mathrm{ml}(A)$ or $10 \mathrm{ng} / \mathrm{ml}$ (B) Fg2 monitored in the same field of view at one, two and four days after plating in Matrigel. Note that on day I, just before adding Fg2 to the cells, CPe aggregates in (A) and (B) are similar in size. On day 2, Fgf2-treated CPe cells have formed bigger aggregates that are fewer in number compared to non-treated cells. On day 4, vesicles in the Fgf2-treated culture are larger than in control culture, but fewer in number. (C) Graph showing the average size of aggregates and vesicles in cultures treated without (control) or with $10 \mathrm{ng} / \mathrm{ml}$ Fgf2 at different times after seeding according to a multiple linear regression analysis. The fitted lines show that Fgf-treated aggregates and vesicles become bigger in size over time. The average size difference between control and Fgf2 treated cultures is $462.5 \mu \mathrm{m}^{2}$ (SEM: $87.25 \mu \mathrm{m}^{2}$ ), and Fgf2-treated aggregates increase every day on average by $492.03 \mu \mathrm{m}^{2}$ (compared to control aggregates. (D) Bar chart showing the mean number of aggregates and vesicles in cultures treated without or with $10 \mathrm{ng} / \mathrm{ml}$ Fgf2. At day 2 and day 4 after seeding, the number of aggregates and vesicles is significantly lower in Fgf2-treated cultures compared to controls $(p<0.05)$, whereas before the addition of Fgf2 on day I, the difference is not significant. Data are means \pm SEM.
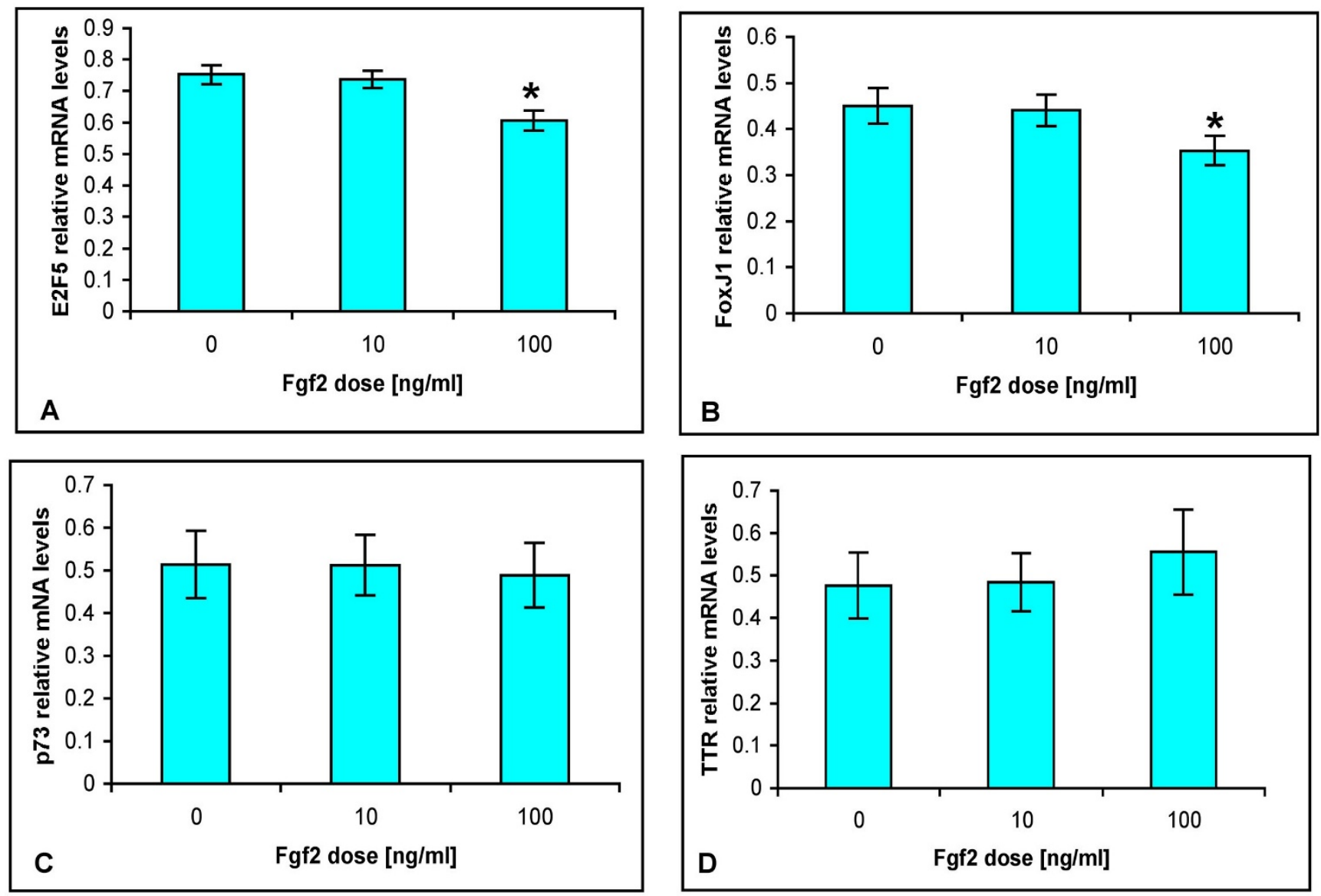

Figure 6

Effect of Fgf2 on the expression of genes associated with CPe development and function in TRCSFB-2 cells. The bar charts show the average level of mRNA expression for A: E2f5 ( $\mathrm{n} \geq 9)$, B: FoxJI ( $\geq 12)$, C: p73 ( $\geq 7)$ and D: TTR ( $\geq 1 \mathrm{I}$ ) in response to increasing Fgf2 concentrations $(0 \mathrm{ng} / \mathrm{ml}, 10 \mathrm{ng} / \mathrm{ml}, 100 \mathrm{ng} / \mathrm{ml})$. Expression of each gene was assessed by RT-PCR and normalised against expression of the housekeeping gene, GAPDH. Both E2f5 and FoxJI transcripts are significantly reduced by treatment with $100 \mathrm{ng} / \mathrm{ml}(p<0.05)$. Data are means \pm SEM. 
tested, $100 \mathrm{ng} / \mathrm{ml}$, (Fig 6A-B), but TTR and $p 73$ expression was not significantly affected at any Fgf2 concentration (Fig 6C-D). These results suggest that Fgf2 may play a role in modulating CPe function.

\section{Discussion \\ Fgf2 expression is an early event in CPe development} It was shown here that Fgf2 is expressed not only in mature CPe as previously reported [27,28], but also at early embryonic stages, and that such expression is conserved between mouse and human. As FgfRs are also expressed in the developing choroid plexus in both species $[26,34]$, it is likely that Fgf2 can behave in an autocrine fashion in vivo, consistent with its ability to respond to Fgf2 in vitro demonstrated in this study.

Analysis of Fgf2 expression, cell division during development, and the effect of Fgf2 on CP cells cultured in Matrigel suggest that this growth factor does not play a key role in $\mathrm{CPe}$ growth. Whilst the concentrations of the growth factor used here (mainly $10 \mathrm{ng} / \mathrm{ml}$ ) and in many in vitro studies may seem high in comparison to that in CSF in vivo [35], it is the concentration of Fgf2 at the cell surface which is critical [12,36-38]. As discussed above, autocrine and paracrine Fgf signalling is likely to occur in the $\mathrm{CPe}$, and Fgf2 availability is regulated by the extracellular matrix. Although in our 3D culture system the bioavailability of Fgf2 may be modified as a result of interactions with the extracellular matrix, a clear effect on vesicle size was observed at all the concentrations tested.

Whereas CP cells in Matrigel are not induced to proliferate in response to Fgf2, CP cells grown as monolayers, either on laminin or collagen, significantly increase their proliferative activity in the presence of low/medium Fgf2 concentrations. This different proliferative response may be largely due to the differences in culture architecture in the two models. CPe morphology is different in these two culture conditions, with cells being initially rounded and then tightly packed to form a simple polarized epithelium in Matrigel, and well-spread and flat in monolayers. An environment-dependent proliferative effect of Fgf2 on $\mathrm{CPe}$ cells is also consistent with previous work showing that these cells behave differently when plated on different matrices [39]. These authors tested growth factors present in trace amounts in Matrigel, Tgf $\beta 1, \operatorname{Tgf} \beta 2$ and Pdgf, for their ability to increase penetration of CPe into matrices, and suggested that $\operatorname{Tgf} \beta 2$, but not $\operatorname{Tgf} \beta 1$ and Pdgf, can increase migration depending on matrix composition. A role of the environment in Fgf2-induced proliferative response is also consistent with in vivo studies reporting increased proliferation in Fgf2-treated CPe in pathological conditions, such as in a Parkinson's disease model [23], but no obvious size changes in the CPe of normal animals that developed Fgf2-induced hydrocephalus [23].

Fgf2 increases CPe cell aggregation rather than secretion It has been previously shown that growth of CPe vesicles depends on fluid secretion within the lumen [30]. Analysis of vesicle growth rate has shown that Fgf2-induced increase in vesicle size is not due to increased secretion into the lumen. The slopes of the growth curves of vesicles exposed to different Fgf2 concentrations are very similar, and no increase in the rate of recovery of vesicles collapsed using secretion inhibitors is induced by Fgf 2 treatment. In addition, if Fgf2 treatment is started at 6 days, once the vesicles have already formed, no increase in vesicle size is observed in treated cultures 4 days later. Transmission electron microscopy analysis of Fgf2-treated vesicles (not shown) is also consistent with lack of significant Fgf2 effects on secretion, as no obvious reduction in electron density, which is thought to decrease in CPe with very high secretory activity, is observed in these vesicles. Our results in vitro are consistent with work suggesting that the occurrence of hydrocephalus observed in response to Fgf2 treatment in vivo is not caused by a direct effect of Fgf2 on CPe secretion, but could be due, at least in part, to a decrease in CSF absorption caused by impaired function of the arachnoid villi, the main structure involved in this process [23].

Fgf2-induced vesicle growth is due to increased CPe cell recruitment into the aggregates that form vesicles, as indicated by a reduced number of aggregates and increase in aggregate size in the presence of Fgf2. The mechanisms underlying increased cell recruitment leading to formation of larger aggregates have yet to be fully elucidated, but the present results suggest an increase in cell-cell adhesion rather than in cell motility. Fgf2-treated aggregates appear more compact than controls. Time-lapse microscopy analysis has not indicated any obvious increase in cell motility in the presence of Fgf2 and, as discussed earlier, proliferation does not appear to contribute to increased aggregate size. E-cadherin could be a candidate mediator of the effect of Fgf in the CPe, as it is expressed in adult CP and Fgf treatment can upregulate E-cadherin expression in pancreatic cancer cell lines [40-42]. A thorough adhesion molecule profile of the CPe is required to identify putative Fgf targets leading to increased cell aggregation.

The fact that Fgf2 is expressed early during choroid plexus development and appears to affect CPe cell-cell interaction, rather than cell proliferation, is consistent with a role of Fgf2 in maintaining the integrity of this epithelium as recently suggested for endothelial cells of the blood brain barrier [36]. 


\section{Fgf2 modulates expression of transcription factors associated with CPe homeostasis}

The effect of Fgf2 on E2f5 and Foxj1 is of particular interest, given the putative roles of these transcription factors in the CPe. Knockouts of E2f5, a member of a family (E2f1-E2f6) of transcriptional regulators and FoxJ1, a member of the forkhead-box (Fox)/winged helix gene family, have been associated with defective choroid plexus function resulting in hydrocephalus [4-6]. Foxj1 is associated with ciliogenesis and maturation of $\mathrm{CPe}$ $[4,9,43]$, and Fgf2-induced down-regulation of Foxj1 has been reported in other cell types [12]. In addition, defects in cilia formation have been recently reported in Fgf10 null mutants, supporting the view that Fgf signalling may play a role in ciliogenesis [44]. E2f5 is a general cell cycle regulator, but in the $\mathrm{CPe}$ seems associated with $\mathrm{CPe}$ function rather than proliferation [5,7]. Since E2f5 intracellular localization changes with CPe maturation, and the FgfRs are differentially regulated during its development $[7,26]$, it is conceivable that Fgf signalling may play a role in modulating translocation of this protein from the nucleus to the cytoplasm in the developing CPe.

The dose-dependent effects of Fgf2 on proliferation (induced at low/medium concentrations), and gene expression (induced at high concentrations) in the $\mathrm{CPe}$ cell line are akin to those observed in other cell types, such as fibroblasts and cranial neural crest cells, where at lower concentrations Fgf2 stimulates proliferation, whereas at higher concentrations it appears to support survival and differentiation $[37,45]$.

\section{Conclusion}

Altogether, this study suggests that whereas Fgf2 does not have a significant direct effect on CSF secretion, it is an endogenous regulator of the Fgf signalling pathway in the developing choroid plexus, given its conserved expression in rodents and humans. Furthermore, Fgf2 is likely to play a role in maintaining $\mathrm{CPe}$ integrity and function, as suggested by the increase in CP cell aggregation and modulation of gene expression observed in vitro. In order to better understand the role of Fgf2 on cell-cell interaction it will be important to identify which cell-cell adhesion molecules might be modulated by Fgf2 in the CPe either at the mRNA or protein level and other Fgf ligands that may be crucial for Fgf signalling in the CPe.

\section{Competing interests}

The authors declare that they have no competing interests.

\section{Authors' contributions}

SG and AS carried out the experimental work, contributed to its design, interpretation of the results and to the first draft of the manuscript. AW advised on the statistical analysis and supervised SG and AS on this aspect of the work.
TT developed the cell line used in this study and gave final approval to the manuscript. PF conceived, designed and funded the study, carried out data analysis and interpretation of the results, and finalized the manuscript. All authors have read and approved the final version of the manuscript.

\section{Additional material}

\section{Additional file 1}

Time-lapse of control CPe vesicle. Two days time-lapse of control CPe vesicle cultures which had been grown for 8 days in Matrigel.

Click here for file

[http://www.biomedcentral.com/content/supplementary/17438454-5-20-S1.mov]

\section{Additional file 2}

Time-lapse of Fgf2-treated CPe vesicle. CPe vesicles grown in the presence of $10 \mathrm{ng} / \mathrm{ml} \mathrm{Fgf2.} \mathrm{Pictures} \mathrm{of} \mathrm{the} \mathrm{same} \mathrm{field} \mathrm{of} \mathrm{view} \mathrm{were} \mathrm{taken} \mathrm{at} 1 \mathrm{~h}$ intervals. Both treated and untreated vesicles are highly pulsatile.

Click here for file

[http://www.biomedcentral.com/content/supplementary/17438454-5-20-S2.mov]

\section{Acknowledgements}

This paper is dedicated to the memory of Peter Thorogood, with whom this work started. We are grateful to Andrew Copp and Jane Sowden, Institute of Child Health, UCL, UK, for useful discussions. This work was supported by the Child Research Appeal Trust and the Great Ormond Street Hospital for Children NHS Trust/Institute of Child Health Science Development Initiative.

\section{References}

I. Redzic ZB, Segal MB: The structure of the choroid plexus and the physiology of the choroid plexus epithelium. Adv Drug Deliv Rev 2004, 56(I 2): 1695-1716.

2. Redzic ZB, Preston JE, Duncan JA, Chodobski A, Szmydynger-Chodobska J: The choroid plexus-cerebrospinal fluid system: from development to aging. Curr Top Dev Biol 2005, 7 I: I-52.

3. Johansson PA, Dziegielewska KM, Liddelow SA, Saunders NR: The blood-CSF barrier explained: when development is not immaturity. Bioessays 2008, 30:237-248.

4. Chen J, Knowles HJ, Hebert JL, Hackett BP: Mutation of the mouse hepatocyte nuclear factor/forkhead homologue 4 gene results in an absence of cilia and random left-right asymmetry. J Clin Invest 1998, I02:1077-1082.

5. Lindeman G], Dagnino L, Gaubatz S, Xu Y, Bronson RT, Warren HB, Livingston DM: A specific, nonproliferative role for E2F-5 in choroid plexus function revealed by gene targeting. Genes Dev 1998, I 2: 1092-1098.

6. Yang A, Walker N, Bronson R, Kaghad M, Oosterwegel M, Bonnin J, Vagner C, Bonnet H, Dikkes P, Sharpe A, McKeon F, Caput D: p73deficient mice have neurological, pheromonal and inflammatory defects but lack spontaneous tumours. Nature 2000 , 404:99-103.

7. Swetloff A, Ferretti P: Changes in E2F5 intracellular localization in mouse and human choroid plexus epithelium with development. Int J Dev Biol 2005, 49:859-865.

8. Dagnino L, Fry CJ, Bartley SM, Farnham P, Gallie BL, Phillips RA: Expression patterns of the E2F family of transcription factors during mouse nervous system development. Mech Dev 1997, 66:13-25.

9. Lim L, Zhou H, Costa RH: The winged helix transcription factor HFH-4 is expressed during choroid plexus epithelial develop- 
ment in the mouse embryo. Proc Natl Acad Sci USA 1997, 94:3094-3099.

10. Cabrera-Socorro A, Pueyo Morlans M, Suarez Sola ML, Gonzalez Delgado FJ, Castaneyra-Perdomo A, Marin MC, Meyer G: Multiple isoforms of the tumor protein p73 are expressed in the adult human telencephalon and choroid plexus and present in the cerebrospinal fluid. Eur I Neurosci 2006, 23:2109-2 II8.

II. Meyer G, Cabrera Socorro A, Perez Garcia CG, Martinez Millan L, Walker N, Caput D: Developmental roles of p73 in Cajal-Retzius cells and cortical patterning. J Neurosci 2004, 24:9878-9887.

12. Yoshida E, Atkinson TG, Chakravarthy B: Neuroprotective gene expression profiles in ischemic cortical cultures preconditioned with IGF-I or bFGF. Brain Res Mol Brain Res 2004, | 31:33-50.

13. Liszczak TM, Black PM, Foley L: Arginine vasopressin causes morphological changes suggestive of fluid transport in rat choroid plexus epithelium. Cell Tissue Res 1986, 246:379-385.

14. Johanson CE, Preston JE, Chodobski A, Stopa EG, Szmydynger-Chodobska J, McMillan PN: AVP VI receptor-mediated decrease in $\mathrm{Cl}$-efflux and increase in dark cell number in choroid plexus epithelium. Am J Physiol 1999, 276:C82-90.

15. Johanson CE, Donahue JE, Spangenberger A, Stopa EG, Duncan JA, Sharma HS: Atrial natriuretic peptide: its putative role in modulating the choroid plexus-CSF system for intracranial pressure regulation. Acta Neurochir 2006, 96:45I-456.

16. Nilsson C, Hultberg BM, Gammeltoft S: Autocrine role of insulinlike growth factor II secretion by the rat choroid plexus. Eur J Neurosci 1996, 8:629-635.

17. Rudman D, Hollins BM, Lewis NC, Scott JW: Effects of hormones on 3', 5'-cyclic adenosine monophosphate in choroid plexus. Am J Physiol 1977, 232:E353-357.

18. Szebenyi G, Fallon JF: Fibroblast growth factors as multifunctional signaling factors. Int Rev Cytol 1999, 185:45-106.

19. Vaccarino FM, Schwartz ML, Raballo R, Nilsen J Rhee J, Zhou M, Doetschman T, Coffin JD, Wyland JJ, Hung YT: Changes in cerebral cortex size are governed by fibroblast growth factor during embryogenesis. Nature Neurosci 1999, 2:246-253.

20. Kuhn HG, Winkler J, Kempermann G, Thal LJ, Gage FH: Epidermal growth factor and fibroblast growth factor-2 have different effects on neural progenitors in the adult rat brain. J Neurosci 1997, 1 7:5820-5829

21. Yoshimura S, Takagi Y, Harada J, Teramoto T, Thomas SS, Waeber C, Bakowska JC, Breakefield XO, Moskowitz MA: FGF-2 regulation of neurogenesis in adult hippocampus after brain injury. Proc Natl Acad Sci USA 200I, 98:5874-5879.

22. Pearce RK, Collins P, Jenner P, Emmett C, Marsden CD: Intraventricular infusion of basic fibroblast growth factor (bFGF) in the MPTP-treated common marmoset. Synapse 1996, 23:192-200

23. Johanson CE, Szmydynger-Chodobska J, Chodobski A, Baird A, McMillan P, Stopa EG: Altered formation and bulk absorption of cerebrospinal fluid in FGF-2-induced hydrocephalus. Am J Physiol 1999, 277:R263-27I.

24. Ohmiya M, Fukumitsu H, Nitta A, Nomoto H, Furukawa $Y$, Furukawa S: Administration of FGF-2 to embryonic mouse brain induces hydrocephalic brain morphology and aberrant differentiation of neurons in the postnatal cerebral cortex. Neurosci Res 200I, 65:228-235.

25. Alzheimer $C$, Werner S: Fibroblast growth factors and neuroprotection. Adv Exp Med Biol 2002, 5 | 3:335-35।.

26. Reid S, Ferretti P: Differential expression of FGFRs in the developing murine choroid plexus. Brain Res Dev 2003, 141( ( \&\#452): 15-24.

27. Cuevas P, Carceller F, Reimers D, Fu X, Gimenez-Gallego G: Immunohistochemical localization of basic fibroblast growth factor in choroid plexus of the rat. Neurol Res 1994, 16:310-312.

28. Fuxe K, Tinner B, Zoli M, Pettersson RF, Baird A, Biagini G, Chadi G, Agnati LF: Computer-assisted mapping of basic fibroblast growth factor immunoreactive nerve cell populations in the rat brain. I Chem Neuroanat 1996, I I:I 3-35.

29. Raballo R, Rhee J, Lyn-Cook R, Leckman JF, Schwartz ML, Vaccarino FM: Basic fibroblast growth factor (Fgf2) is necessary for cell proliferation and neurogenesis in the developing cerebral cortex. J Neurosci 2000, 20:5012-5023.
30. Swetloff A, Greenwood S, Wade AM, Ferretti P: Growth of choroid plexus epithelium vesicles in vitro depends on secretory activity. J Cell Physiol 2006, 208:549-555.

31. Thomas T, Stadler E, Dziadek M: Effects of the extracellular matrix on fetal choroid plexus epithelial cells: changes in morphology and multicellular organization do not affect gene expression. Exp Cell Res 1992, 203:198-213.

32. Terasaki T, Hosoya K: Conditionally immortalized cell lines as a new in vitro model for the study of barrier functions. Biol Pharm Bull 2001, 24: III-II8.

33. Goldstein H: Multilevel statistical models. Kendall's Library of Statistics 3. 2nd edition. London: Hodder Arnold Publishing: 1995.

34. Chan CT, Thorogood P: Pleiotropic features of syndromic craniosynostoses correlate with differential expression of fibroblast growth factor receptors $I$ and 2 during human craniofacial development. Pediatr Res 1999, 45:46-53.

35. Malek AM, Connors S, Robertson RL, Folkman J, Scott RM: Elevation of cerebrospinal fluid levels of basic fibroblast growth factor in moyamoya and central nervous system disorders. Pediat Neurosur 1997, 27(2): 182-189.

36. Bendfeldt K, Radojevic V, Kapfhammer J, Nitsch C: Basic fibroblast growth factor modulates density of blood vessels and preserves tight junctions in organotypic cortical cultures of mice: a new in vitro model of the blood-brain barrier. J Neurosci 2007, 27:3260-3267.

37. Garcia-Maya M, Anderson AA, Kendal CE, Kenny AV, EdwardsIngram LC, Holladay A, Saffell JL: Ligand concentration is a driver of divergent signaling and pleiotropic cellular responses to FGF. J Cell Physiol 2006, 206:386-393.

38. Trudel C, Faure-Desire V, Florkiewicz RZ, Baird A: Translocation of FGF2 to the cell surface without release into conditioned media. J Cell Physiol 2000, 185:260-268.

39. Stadler E, Dziadek M: Extracellular matrix penetration by epithelial cells is influenced by quantitative changes in basement membrane components and growth factors. Exp Cell Res 1996, 229:360-369.

40. Figarella-Branger D, Lepidi H, Poncet C, Gambarelli D, Bianco N, Rougon G, Pellissier JF: Differential expression of cell adhesion molecules (CAM), neural CAM and epithelial cadherin in ependymomas and choroid plexus tumors. Acta Neuropathol (Berl) 1995, 89:248-257.

4I. Napolitano EW, Venstrom K, Wheeler EF, Reichardt LF: Molecular cloning and characterization of B-cadherin, a novel chick cadherin. J Cell Biol I99I, I I 3:893-905.

42. El-Hariry I, Pignatelli M, Lemoine NR: FGF-I and FGF-2 regulate the expression of $\mathrm{E}$-cadherin and catenins in pancreatic adenocarcinoma. Int I Cancer 200I, 94:652-66I.

43. Brody SL, Yan XH, Wuerffel MK, Song SK, Shapiro SD: Ciliogenesis and left-right axis defects in forkhead factor HFH-4-null mice. Am J Respir Cell Mol Biol 2000, 23:45-5I.

44. Pauley S, Wright T], Pirvola U, Ornitz D, Beisel K, Fritzsch B: Expression and function of FGFIO in mammalian inner ear development. Dev Dyn 2003, 227:203-215.

45. Sarkar S, Petiot A, Copp A, Ferretti P, Thorogood P: FGF2 promotes skeletogenic differentiation of cranial neural crest cells. Development 200I, 128:2143-2152.

Publish with Bio Med Central and every scientist can read your work free of charge

"BioMed Central will be the most significant development for disseminating the results of biomedical research in our lifetime. "

Sir Paul Nurse, Cancer Research UK

Your research papers will be:

- available free of charge to the entire biomedical community

- peer reviewed and published immediately upon acceptance

- cited in PubMed and archived on PubMed Central

- yours - you keep the copyright 